\section{ESTEATOSE HEPÁTICA NÃO ALCOÓLICA: RELAÇÃO COM A SÍNDROME METABÓLICA E OS FATORES DE RISCO CARDIOVASCULAR EM ADOLESCENTES OBESOS}

\author{
Nonalcoholic hepatic steatosis: association with metabolic \\ syndrome and cardiovascular risk factors in obese adolescents
Esteatosis hepática no alcohólica: relación con el síndrome metabólico y los factores de riesgo cardiovascular en adolescentes obesos

Artigo Original

\title{
RESUMO
}

Objetivo: Avaliar a associação entre esteatose hepática não alcoólica (EHNA), síndrome metabólica (SM) e fatores de risco cardiovascular (FRCs) em adolescentes obesos. Métodos: Estudo observacional do tipo transversal com abordagem quantitativa, realizado de junho a agosto de 2011. Os voluntários foram aleatoriamente selecionados e encaminhados para avaliação clínica no setor de endocrinologia e cardiologia do hospital de clínicas da Universidade Federal de Uberlândia. Participaram 34 adolescentes de 14 a 19 anos, acima do percentil 95 da curva de crescimento. A EHNA foi analisada por ultrassonografia. A SM e os FRCs foram identificados pelos critérios da International Diabetes Federation. Resultados: A amostra foi composta de 14 indivíduos do sexo masculino e 20 do sexo feminino, com idade $(16,8 \pm 1,6)$ e índice de massa corporal (IMC) $(35,7 \pm 3,9)$. A ocorrência de EHNA e SM foi de $76,5 \%(n=26)$ e $50 \%(n=17)$, respectivamente. O sexo masculino apresentou maior ocorrência de EHNA $(78,6 \%, n=11), \operatorname{SM}(64,3 \%, n=09)$ e associação de EHNA com SM (50\%, n=07). Em relação aos FRCs 100\% $(n=34), 61,8 \%(n=21)$ e 52,9\% $(n=18)$ dos adolescentes apresentaram valores aumentados da circunferência da cintura (CC), da lowdensity-lipoprotein cholesterol (LDL-C) e pressão arterial sistólica (PAS), respectivamente, e $52,9 \%(\mathrm{n}=18)$ apresentaram baixos valores de high-density lipoprotein cholesterol (HDL-C). Houve correlações entre SM e triglicerídeos, PAS e diastólica e HDL-C, e entre EHNA, IMC e CC. Conclusão: Observou-se uma alta ocorrência de EHNA, SM e FRCs em adolescentes obesos. Uma forte correlação foi observada entre EHNA e SM, e entre FRCs com EHNA e SM.

Descritores: Adolescentes; Obesidade Infantil; Fígado Gorduroso; Síndrome X Metabólica; Doenças Cardiovasculares.

\section{ABSTRACT}

Objective: To evaluate the association between nonalcoholic hepatic steatosis (NAHS), metabolic syndrome (MS) and cardiovascular risk factors (CRF) in obese adolescents. Methods: Cross-sectional observational study with a quantitative approach, carried out from June to August 2011. The volunteers were randomly selected and referred to clinical evaluation in the endocrinology and cardiology units at the clinics hospital of the Federal University of Uberlandia, being included 34 adolescents of 14-19 years above the 95th percentile of the growth curve. NAHS was assessed by ultrasonography. The MS and CRF were diagnosed by the International Diabetes Federation criteria. Results: The sample consisted of 14 male and 20 female patients aged $16.8 \pm 1.6$ and body mass index (BMI) of $35.7 \pm 3.9$. The occurrence of NAHS and MS was $76.5 \%$ and $50 \%$, respectively. Males had a higher incidence of NAHS (78.6\%), SM (64.3\%) and association of NAHS with MS (50\%). Regarding the CRF, $100 \%(n=34), 61.8 \%(n=21)$ and $52.9 \%(n=18)$ of the adolescents had elevated values of waist circumference (WC), low-density lipoprotein cholesterol (LDL-C)

\author{
Wener Barbosa Resende ${ }^{(1)}$ \\ Carlos Eduardo Caetano ${ }^{(1)}$ \\ Ana Maria Oliveira Caixeta \\ Nogueira ${ }^{(2)}$ \\ Sandra Regina Xavier Santos ${ }^{(1)}$ \\ Rafael Dias da Costa e Silva ${ }^{(3)}$ \\ João Elias Nunes ${ }^{(1)}$ \\ Heitor Santos Cunha ${ }^{(1)}$ \\ Nadia Carla Cheik ${ }^{(1)}$
}

1) Universidade Federal de Uberlândia UFU - Uberlândia (MG) - Brasil

2) Secretaria Municipal de Saúde de Uberlândia - SMSU - Uberlândia (MG) -

Brasil

3) DCS Diagnósticos por Imagem - Birigui (SP) - Brasil

Recebido em: 01/04/2013 Revisado em: 02/06/2013 Aceito em: 02/01/2014 
and systolic blood pressure (SBP), respectively, and $52.9 \%$ $(n=18)$ showed low levels of high-density lipoprotein cholesterol (HDL-C). There were correlations between MS and triglycerides; systolic and diastolic blood pressure and HDL-C; and between NAHS and BMI and WC. Conclusion: A high occurrence of NAHS, SM and CRF was observed in obese adolescents. A strong correlation was observed between MS and NAHS, and between FRC and NAHS and SM.

Descriptors: Adolescents; Obesity; Fatty Liver; Metabolic Syndrome X; Cardiovascular Diseases.

\section{RESUMEN}

Objetivo: Evaluar la asociación entre la esteatosis hepática no alcohólica (EHNA), síndrome metabólico (SM) y factores de riesgo cardiovascular (FRCs) en adolescentes obesos. Métodos: Estudio observacional del tipo trasversal con abordaje cuantitativo realizado entre junio y agosto de 2011. Los voluntarios fueron seleccionados al azar y encaminados para evaluación clínica en el sector de endocrinología y cardiología del hospital de la Universidad Federal de Uberlandia. Participaron 34 adolescentes entre 14 y 19 años que estaban por encima del percentil 95 de la curva de crecimiento. La EHNA fue analizada con la ultrasonografía. La SM y los FRCs fueron identificados a través de criterios de la International Diabetes Federtion. Resultados: La muestra fue constituida de 14 individuos del sexo masculino y 20 del sexo femenino con media de edad de 16,8 8 1,6

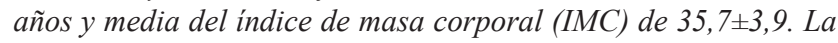
ocurrencia de EHNA y SM fue del 76,5\% $(n=26)$ y 50\% $(n=17)$, respectivamente. El sexo masculino presentó mayor ocurrencia de EHNA (78,6\%, $n=11), S M(64,3 \%, n=09)$ y asociación de EHNA y SM $(50 \%, n=07)$. Respecto a los FRCs, el 100\% $(n=34), 61,8 \%$ $(n=21)$ y el $52,9 \%(n=18)$ de los adolescentes presentaron valores mayores de la circunferencia de cintura (CC), de la low-densitylipoprotein cholesterol (LDL-C) y tensión arterial sistólica (TAS), respectivamente, y el 52,9\% $(n=18)$ presentó bajos niveles de high-density lipoprotein cholesterol (HDL-C). Hubieron correlaciones entre SM y triglicérides, TAS y diastólica y HDL-C, y entre EHNA, IMC y CC. Conclusión: Se observó una elevada ocurrencia de EHNA, SM y FRCs en adolescentes obesos. Una fuerte correlación fue observada entre EHNA y SM, y entre FRCs y EHNA y SM.

Descriptores: Adolescente; Obesidad Pediátrica; Hígado Gras; Síndrome X Metabólico; Enfermedades Cardiovasculares.

\section{INTRODUÇÃO}

A doença hepática gordurosa não alcoólica (DHGNA) abrange todo o espectro de doença do fígado gorduroso em indivíduos sem consumo significativo de álcool, variando de esteatose hepática até esteato-hepatite e cirrose, sendo considerada como a principal causa de doença crônica do fígado em adultos e na população pediátrica ${ }^{(1,2)}$.
A esteatose hepática não alcoólica (EHNA) em crianças é associada com obesidade, resistência à insulina (RI), síndrome metabólica (SM) e seus componentes são fatores de risco cardiovascular $(\mathrm{CV})^{(3,4)}$.

Essa doença tem alcançado proporções epidêmicas nos países ocidentais. Nos Estados Unidos estima-se que aproximadamente $20 \%$ da população adulta tenham alguma manifestação de EHNA. A prevalência aumenta na população obesa, variando de $75 \%$ a $92 \%$. Em crianças e adolescentes, essa prevalência varia de $9,6 \%$ a $14 \%$ aumentando para $38 \%$ em indivíduos obesos. Valores similares já são encontrados em países da Europa e Ásia ${ }^{(5,6)}$. No Brasil, ainda não há dados consistentes sobre a prevalência de EHNA em adultos e na população pediátrica. No entanto, estudos tem mencionado valores que variam de $13,5 \%$ a $41,7 \%$ na população adulta e ocorrência de $2,6 \%$ e $57,4 \%$ em crianças eutróficas e obesas, respectivamente ${ }^{(7-9)}$.

A associação da EHNA com a SM sugere que a EHNA pode ser um componente hepático da SM que representa um emergente problema de saúde em diferentes populações étnicas no mundo ${ }^{(10,11)}$. Em crianças e adolescentes torna-se um precursor de diabetes mellitus e doenças cardiovasculares na vida adulta ${ }^{(3,12)}$.

A prevalência da SM na população pediátrica modificase de acordo com a população estudada e com o critério utilizado para avaliação. No entanto, estudos recentes demonstram uma ocorrência que varia de $4,2 \%$ a $38,7 \%$ em ambos os $\operatorname{sexos}^{(12,13)}$. Na população brasileira, a literatura tem apresentado uma variação de $15,6 \%$ a $49,1 \%^{(11,14)}$. Em pessoas jovens obesas representa um problema para saúde pública, especialmente considerando a idade pós-púbere, quando a obesidade tem um grande risco de permanecer até a idade adulta ${ }^{(14)}$. Em 10 anos, a prevalência da obesidade e da SM aumentará consideravelmente nos indivíduos em transição de faixa etária (de crianças e adolescentes para adultos jovens), de $21 \%$ para $33,4 \%$ e de $2,8 \%$ para $17,9 \%$, respectivamente ${ }^{(15)}$.

Além disso, no Brasil, o número de adolescentes do sexo masculino entre 10 a 19 anos idade com excesso de peso passou de 3,7\% (1974-1975) para 21,7\% (2008-2009), e o número de adolescentes do sexo feminino com excesso de peso passou de 7,6\% para 19,4\%, no mesmo período, segundo a Pesquisa de Orçamento Familiar (POF) ${ }^{(16)}$.

Sendo assim, o presente estudo objetivou avaliar a associação entre esteatose hepática não alcoólica, síndrome metabólica e fatores de risco cardiovascular em adolescentes obesos. Os resultados do presente estudo servirão de respaldo para elaboração de estratégias de promoção da saúde e prevenção e controle de doenças dessa população e assim minimizar o surgimento de doenças crônicas não transmissíveis em adolescentes com excesso de peso. 


\section{MÉTODOS}

Este estudo caracterizou-se como observacional do tipo transversal com abordagem quantitativa, realizado de junho a agosto de 2011 na cidade de Uberlândia-Minas Gerais (MG).

A amostra foi constituída por adolescentes de 14 a 19 anos de idade, recrutados a partir da divulgação em mídia eletrônica e impressa. Posteriormente, os adolescentes obesos foram encaminhados para o setor de endocrinologia e cardiologia da Universidade Federal de Uberlândia (SECHC-UFU) para avaliação clínica.

De um total de 175 adolescentes encaminhados para o SECHC-UFU, 34 adolescentes (14 do sexo masculino e 20 do sexo feminino) foram selecionados por amostragem probabilística aleatória simples, enquadrando-se nos critérios de inclusão: índice de massa corporal (IMC) acima do percentil 95 da curva de crescimento do Centers for Disease Control (CDC) ${ }^{(17)}$ e com maturação sexual ${ }^{(18,19)}$. Excluíram-se os adolescentes com hábitos etílicos e tabágicos, e portadores de doenças incapacitantes, como: reumáticas, neuromusculares, osteoarticulares ou degenerativas informadas por relatório médico.

Os adolescentes foram pesados usando roupas leves e sem calçados em uma balança Filizola ${ }^{\circledR}$ com capacidade para $150 \mathrm{~kg}$ e sensibilidade de $100 \mathrm{~g}$, a estatura foi obtida utilizando-se um estadiômetro de madeira da marca Sanny ${ }^{\circledR}$, com escala de precisão de $0,1 \mathrm{~cm}$. O índice de massa corporal (IMC) foi calculado dividindo-se a massa corporal $(\mathrm{kg})$ pelo quadrado da estatura $(\mathrm{m})$.

Analisou-se a presença de esteatose, as medidas da gordura visceral (GV) e a gordura subcutânea (GSC) por ultrassom. Determinou-se o aspecto ultrassonográfico da esteatose pelo aumento difuso da ecogenicidade hepática, que pode ser notada comparando-se a ecogenicidade do fígado com a do córtex renal ou do baço ${ }^{(20)}$. De acordo com o diagnóstico, classificou-se a esteatose em graus: leve (I), moderado (II) e severo (III) ${ }^{(21)}$.

Realizou-se a medida da GV com transdutor convexo de $3,5 \mathrm{MHz}$ posicionado transversalmente a $1,0 \mathrm{~cm}$ acima da cicatriz umbilical, e considerada como a medida entre a face interna do músculo reto abdominal e a parede anterior da aorta na linha média do abdome, quantificada em centímetros ${ }^{(22)}$. A medida da GSC foi realizada com transdutor linear de $7,5 \mathrm{MHz}$ posicionado transversalmente a 1,0 cm da cicatriz umbilical, sendo considerada a medida entre a pele e a face externa da fáscia do músculo reto do abdome, quantificada em centímetro ${ }^{(22)}$.

Para caracterização da síndrome metabólica nos adolescentes, utilizou-se o critério proposto pela International Diabetes Federation (IDF) ${ }^{(23)}$, considerando que para adolescentes de 10 a 16 anos, a síndrome metabólica pode ser diagnosticada pela presença de obesidade abdominal e mais duas alterações (hipertrigliceridemia, baixos níveis de high-density lipoprotein cholesterol (HDL-C), hipertensão arterial sistêmica e hiperglicemia de jejum). Para os adolescentes maiores de 16 anos aplicou-se os critérios de definição para adultos ${ }^{(23)}$.

Os valores de referência utilizados para adolescentes de 10 a 16 anos foram: circunferência da cintura $(C C) \geq$ que o percentil 90, triglicerídeos $\geq 150 \mathrm{mg} / \mathrm{dL}$, HDL-C $<40 \mathrm{mg} /$ $\mathrm{dL}$, pressão arterial sistólica $\geq 130 \mathrm{e} / \mathrm{ou}$ diastólica $\geq 85 \mathrm{~mm}$ $\mathrm{Hg}$, glicemia de jejum $\geq 100 \mathrm{mg} / \mathrm{dL}^{(23)}$. Para adolescentes maiores que 16 anos foram: circunferência da cintura (CC) $\geq 90 \mathrm{~cm}$ para homens e $\geq 80 \mathrm{~cm}$ para mulheres ${ }^{(24)}$, mais dois fatores: triglicerídeos $\geq 150 \mathrm{mg} / \mathrm{dL}$ ou tratamento específico para essa anormalidade lipídica, HDL-C $<40$ $\mathrm{mg} / \mathrm{dL}$ para rapazes e $<50 \mathrm{mg} / \mathrm{dL}$ para moças ou tratamento específico para HDL-C, pressão arterial sistólica (PAS) $\geq$ 130 e/ou diastólica (PAD) $\geq 85 \mathrm{mmHg}$ ou tratamento para hipertensão previamente diagnosticada e glicemia de jejum $\geq 100 \mathrm{mg} / \mathrm{dL}$ ou diagnóstico prévio de diabetes tipo $2^{(23)}$.

Determinou-se os perfis lipídico e glicídico a partir de coleta de $8 \mathrm{ml}$ de sangue através de venopunção, após jejum de 12 horas noturno com dieta anterior normal. Para a dosagem do perfil lipídico usou-se o método enzimático determinado espectrofotometricamente através do kit da clinical chemistry (Abbott) com exceção do high-density lipoprotein cholesterol (HDL-C) que foi utilizado kit DiaSys Diagnostic Systems GmbH®. Determinou-se o lowdensity-lipoprotein cholesterol (LDL-C) através da fórmula de Friedewald: LDL-C = CT - HDL-C - (Triglicérides/5) ${ }^{(25)}$. Os pontos de corte para o perfil lipídico adotados foram os valores propostos pela III Diretriz Brasileira sobre Dislipidemias $^{(25)}$.

A concentração plasmática de glicose foi mensurada por meio do método enzimático, determinado espectrofotometricamente com kit da clinical chemistry (Abbott). O ponto de corte utilizado foi o estabelecido pelo International Diabetes Federation (IDF) ${ }^{(26)}$.

A circunferência da cintura foi aferida por meio de fita antropométrica inextensível da marca SANY, graduada em milímetros sobre o ponto médio entre a última costela e a crista ilíaca ${ }^{(27)}$.

A normalidade dos dados foi verificada pelo teste de Shapiro-Wilk. O teste T de Student foi utilizado para avaliar a diferenças das médias entre o sexo masculino e feminino. O teste de correlação de Pearson foi aplicado para verificar a presença de relação entre variáveis quantitativas versus quantitativas. As associações existentes entre variáveis qualitativas foram avaliadas pelo teste de Qui-Quadrado ou teste Exato de Fisher quando necessário. Para as análises foram utilizados os softwares SPSS versão 17.0 e BioEstat versão 5.0. Adotou-se nível de significância de 5\% (p $\leq$ $0.05)$. 
O estudo foi aprovado pelo comitê de ética em pesquisa da Universidade Federal de Uberlândia (CEP/ UFU) (nº 498/10). Todos os voluntários e seus pais/ responsáveis receberam informações sobre o estudo, assinando posteriormente o termo de consentimento livre e esclarecido (TCLE) para participação na pesquisa, respeitando a Resolução 196/96 do Conselho Nacional de Saúde.

\section{RESULTADOS}

No presente estudo, dos 175 adolescentes encaminhados à pesquisa, 19,4\% $(\mathrm{n}=34)$ estavam obesos. $\mathrm{Na}$ amostra $(\mathrm{n}=34)$, foi observado que a média do IMC dos voluntários corresponde ao estadiamento de obesidade grau II (Tabela I).

Não foi observada diferença significativa entre os sexos para a variável idade $(\mathrm{M}=16,5 \pm 1,7 ; \mathrm{F}=16,9 \pm 1,5 ; \mathrm{p}=0,42)$, IMC $(M=35,2 \pm 3,9 ; F=35,2 \pm 4,1 ; p=0,97)$ e circunferência da cintura (CC) $(\mathrm{M}=116,3 \pm 9,1 ; \mathrm{F}=112,5 \pm 10,9 ; \mathrm{p}=0,30)$.
Apenas a altura $(\mathrm{M}=174,4 \pm 6,8 ; \mathrm{F}=163,6 \pm 7,3 ; \mathrm{p}=0,0001)$ e a massa corporal (MC) $(\mathrm{M}=08,2 \pm 15,6 ; \mathrm{F}=95,4 \pm 15,1$; $\mathrm{p}=0,018$ ) apresentaram diferença significativa entre os sexos, com maior média no sexo masculino.

Todos os voluntários apresentaram aumento da $\mathrm{CC}$ e $26,5 \%(\mathrm{n}=9), 52,9 \%(\mathrm{n}=18), 32,3 \%(\mathrm{n}=10), 35,3 \%(\mathrm{n}=12)$ e $61,8 \%(\mathrm{n}=21)$ dos adolescentes tinham aumento dos valores de triglicerídeos, PAS, PAD, colesterol total e LDL-C, respectivamente. Além disso, $52,9 \%(\mathrm{n}=18)$ apresentaram valores de HDL-C abaixo dos valores de referência (64,3\% para o sexo masculino e $45 \%$ para o sexo feminino). Apenas $8,8 \%(\mathrm{n}=3)$ apresentaram valores acima do ponto de corte para glicose de jejum.

Metade da amostra estudada apresentou SM ( $\mathrm{n}=17)$ e $82,3 \%(\mathrm{n}=14)$ dos indivíduos que tinham SM apresentaram concomitantemente EHNA (Tabela II). Observou-se uma alta ocorrência de EHNA $(76,5 \%, n=26)$, com maior ocorrência dos casos $(88,5 \%, \mathrm{n}=23)$ nos estágios iniciais

Tabela I - Dados antropométricos, bioquímicos e fisiológicos dos adolescentes obesos. Uberlândia-MG, 2011.

\begin{tabular}{lcccccccccc}
\hline & $\begin{array}{c}\text { IDADE } \\
(\mathbf{a n o s})\end{array}$ & $\begin{array}{c}\text { ALTURA } \\
(\mathbf{m})\end{array}$ & $\begin{array}{c}\text { MC } \\
\mathbf{( k g})\end{array}$ & $\begin{array}{c}\text { IMC } \\
\left(\mathbf{k g} / \mathbf{m}^{2}\right)\end{array}$ & $\begin{array}{c}\mathbf{C C} \\
\mathbf{( c m})\end{array}$ & $\begin{array}{c}\text { GLI } \\
(\mathbf{m g} / \mathbf{d L})\end{array}$ & $\begin{array}{c}\text { TG } \\
(\mathbf{m g} /\end{array}$ & $\begin{array}{c}\text { HDL-C } \\
(\mathbf{m g} / \mathbf{d L})\end{array}$ & $\begin{array}{c}\text { PAS } \\
(\mathbf{m m H g})\end{array}$ & $\begin{array}{c}\text { PAD } \\
(\mathbf{m m H g})\end{array}$ \\
\hline Média & 16,8 & 1,7 & 101,2 & 35,7 & 114,3 & 82,5 & 134,7 & 45,8 & 127,06 & 81.18 \\
DP & 1,6 & 0,1 & 16,4 & 3,9 & 10,2 & 9,7 & 75,9 & 9,2 & 13,82 & 7.18 \\
\hline
\end{tabular}

DP: desvio padrão; MC: massa corporal; IMC: índice de massa corporal; CC: circunferência da cintura; GLI: glicemia de jejum; TG: triglicerídeos; HDL-C: high-density lipoprotein cholesterol; PAS: pressão arterial sistólica; PAD: pressão arterial diastólica.

Tabela II - Distribuição de ocorrência de síndrome metabólica (SM) e de esteatose hepática não alcoólica (EHNA) em relação ao sexo entre os adolescentes obesos. Uberlândia-MG, 2011.

\begin{tabular}{llcccc}
\hline \multirow{2}{*}{ Variável } & \multicolumn{2}{c}{ Masculino $(\mathbf{n = 1 4})$} & \multicolumn{2}{c}{ Feminino $(\mathbf{n}=\mathbf{2 0})$} & $p$ \\
\hline SM & $\mathbf{n}$ & $\mathbf{\%}$ & $\mathbf{n}$ & $\mathbf{\%}$ & $0,2^{*}$ \\
SM + EHNA & 9 & $64,3 \%$ & 8 & $40 \%$ & $0,4^{*}$ \\
\hline
\end{tabular}

*Teste de Qui-Quadrado.

(estágios I e II) da doença, e 53,8\% ( $\mathrm{n}=14)$ dos indivíduos que apresentaram EHNA também tinham SM, (Tabela III). Foi observada associação $(\mathrm{p}=0,02)$ entre SM e EHNA na amostra estudada $(n=34)$.

Após estratificação por sexo, o sexo masculino (n=14) apresentou maior ocorrência SM juntamente com EHNA (Tabela II e III). Não houve associação entre SM e os sexos, EHNA e os sexos, e EHNA e seus graus de estadiamento (Tabelas II e III).

Verificou-se também na amostra $(n=34)$ que houve correlações positivas e negativas entre os fatores de risco cardiovasculares: massa corporal e IMC, CC, PAS, PAD; índice de massa corporal e CA e PAS; circunferência da cintura e PAS e PAD; colesterol total e LDL-C e TG; HDL-C e PAS; PAS e PAD (Tabela IV).

A Figura 1 mostra que os indivíduos com SM ( $\mathrm{n}=17)$ apresentaram relações significativas com os altos valores de triglicerídeos, PAS, PAD e com o baixo valor de HDL-C quando comparados com indivíduos sem SM. Os adolescentes com EHNA ( $\mathrm{n}=26$ ) apresentaram relações com os maiores valores de IMC e de CC quando comparados com os indivíduos sem EHNA. 
Tabela III - Distribuição de ocorrência de esteatose hepática não alcoólica (EHNA) e de síndrome metabólica e estadiamento do grau da doença em relação ao sexo entre os adolescentes obesos. Uberlândia-MG, 2011.

\begin{tabular}{lccccc}
\hline \multirow{2}{*}{ Variável } & \multicolumn{2}{c}{ Masculino $(\mathbf{n = 1 4 )}$} & \multicolumn{2}{c}{ Feminino $(\mathbf{n}=\mathbf{2 0})$} & $\boldsymbol{p}$ \\
\hline EHNA & $\mathbf{n}$ & $\mathbf{\%}$ & $\mathbf{n}$ & $\mathbf{\%}$ & $0,6^{\#}$ \\
EHNA + SM & 11 & $78,6 \%$ & 15 & $75 \%$ & $0,4^{*}$ \\
Grau I & 7 & $50 \%$ & 7 & $35 \%$ & $0,9^{*}$ \\
Grau II & 6 & $42,8 \%$ & 9 & $45 \%$ & $0,4^{\#}$ \\
Grau III & 4 & $28,6 \%$ & 4 & $20 \%$ & $0,6^{\#}$ \\
\hline
\end{tabular}

*Teste de Qui-Quadrado. ${ }^{*}$ Teste Exato de Fisher.

Tabela IV - Correlação entre os fatores de risco cardiovasculares nos adolescentes obesos. Uberlândia-MG, 2011.

\begin{tabular}{lcccccccccc}
\hline & MC & IMC & CC & GLI & TG & HDL-C & PAS & PAD & CT & LDL-C \\
\hline MC & - & $0,74^{* *}$ & $0,85^{* *}$ & $-0,07$ & 0,04 & $-0,32$ & $0,58^{* *}$ & $0,48^{* *}$ & $-0,22$ & $-0,17$ \\
IMC & $0,74^{* *}$ & - & $0,82^{* *}$ & $-0,28$ & $-0,10$ & $-0,05$ & $0,37^{*}$ & 0,26 & $-0,16$ & $-0,11$ \\
CC & $0,85^{* *}$ & $0,82^{* *}$ & - & $-0,22$ & $-0,02$ & $-0,21$ & $0,41^{*}$ & $0,36^{*}$ & $-0,18$ & $-0,12$ \\
GLI & $-0,07$ & $-0,28$ & $-0,22$ & - & $-0,37$ & 0,17 & $-0,09$ & $-0,15$ & $-0,02$ & 0,09 \\
TG & 0,04 & $-0,10$ & $-0,02$ & $-0,37$ & - & $-0,28$ & 0,01 & 0,07 & $0,45^{*}$ & 0,08 \\
HDL-C & $-0,32$ & $-0,05$ & $-0,21$ & 0,17 & $-0,28$ & - & $-0,47^{* *}$ & $-0,27$ & 0,15 & $-0,00$ \\
PAS & $0,58^{* *}$ & $0,37^{*}$ & $0,41^{*}$ & $-0,09$ & 0,01 & $-0,47^{* *}$ & - & $0,71^{* *}$ & $-0,28$ & $-0,17$ \\
PAD & $0,48^{* *}$ & 0,26 & $0,36^{*}$ & $-0,15$ & 0,07 & $-0,27$ & $0,71^{* *}$ & - & $-0,30$ & $-0,29$ \\
CT & $-0,22$ & $-0,16$ & $-0,18$ & $-0,02$ & $0,45^{*}$ & 0,15 & $-0,28$ & $-0,30$ & - & $0,88^{* *}$ \\
LDL-C & $-0,17$ & $-0,11$ & $-0,12$ & 0,09 & 0,08 & $-0,00$ & $-0,17$ & $-0,29$ & $0,88^{* *}$ & - \\
\hline
\end{tabular}

MC: massa corporal; IMC: índice de massa corporal; CC: circunferência da cintura; GLI: glicemia de jejum; TG: Triglicerídeos; HDL-C: high-density lipoprotein cholesterol; PAS: pressão arterial sistólica; PAD: pressão arterial diastólica; CT: colesterol total; LDL-C: low-density lipoprotein cholesterol. Teste de correlação de Pearson. *p<0,05 e **p $<0,01$.

\section{DISCUSSÃO}

No presente estudo foi observado que $19,4 \%$ dos indivíduos encaminhados à pesquisa estavam obesos, com IMC acima do percentil 95 da curva de crescimento, e de acordo com a média do IMC apresentaram grau II de obesidade, que está vinculado com grave risco de comorbidades ${ }^{(28)}$.

$O$ valor percentual de obesidade encontrado na presente pesquisa apresenta-se superior ao percentual $(16,9 \%)$ encontrado nos EUA entre 2009 e $2010^{(29)}$, ao percentual $(14,4 \%)$ encontrado em estudo realizado em crianças e adolescentes brasileiro ${ }^{(30)} \mathrm{e}$ destacadamente superior $(5,9 \%$ em rapazes e $4 \%$ em moças) ao descrito pela Pesquisa de Orçamento Familiar (POF) realizada no Brasil entre 2008 e $2009^{(16)}$.

Os resultados encontrados, juntamente com os de outros pesquisadores ${ }^{(31,32)}$ mostram que a obesidade na população pediátrica tem aumentado drasticamente em um curto período de tempo, sugerindo uma incapacidade, até o presente momento, de reverter esta tendência mundial que resulta em graves consequências para a qualidade de vida e longevidade do ser humano.

As mudanças ambientais associadas com a sociedade moderna são responsáveis pela maior parte da epidemia da obesidade, resultante de um elevado percentual de gordura mantido através de uma homeostase energética anormal ${ }^{(33)}$. As principais causas desse balanço energético positivo são um estilo de vida hipoativo fisicamente e uma alimentação hipercalórica.

Além disso, na população pediátrica o sobrepeso e a obesidade estão relacionados com a puberdade ${ }^{(34)}$, e existe maior probabilidade do adolescente obeso se tornar um adulto obeso ${ }^{(28)}$. Um estudo realizado em crianças e adolescentes brasileiros mostrou que o sobrepeso e a obesidade estão diretamente relacionados com a maturação sexual. Nesse estudo, os autores identificaram que $23,1 \%$ dos avaliados apresentaram sobrepeso ou obesidade, com maior prevalência de excesso de peso nos indivíduos que relataram sinais de maturação sexual, não havendo diferença significativa do excesso de peso entre os sexos ${ }^{(34)}$. Esses resultados são similares aos encontrados no presente 

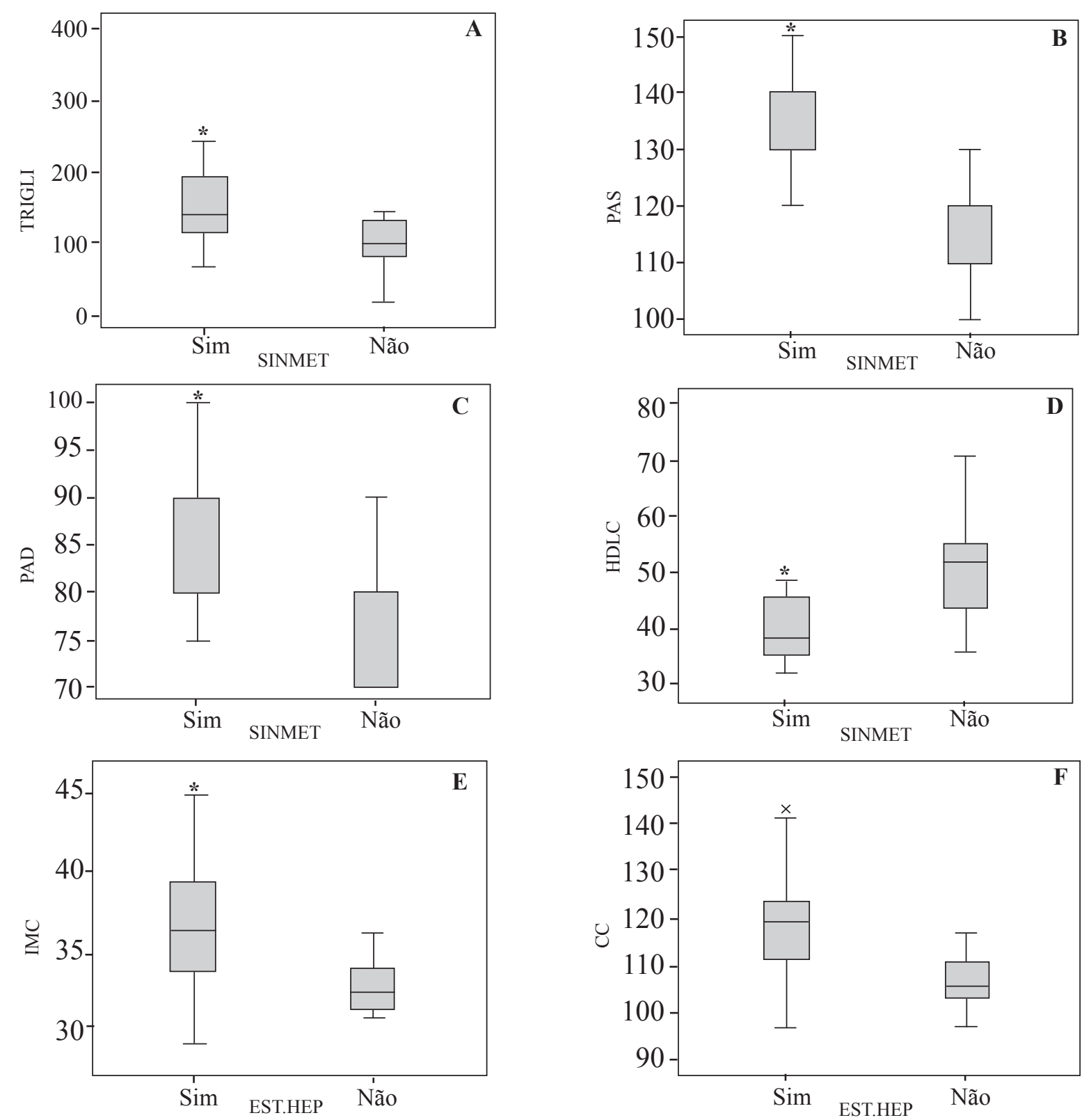

Figura 1 - Relações dos fatores de risco cardiovasculares entre os adolescentes obesos com e sem síndrome metabólica e esteatose hepática não alcoólica. TRIGLI: triglicerídeos em mg/dL; PAS: pressão arterial sistólica em mmHg; PAD: pressão arterial diastólica em mmHg; HDL-C: high-density lipoprotein cholesterol em mg/dL; SÍNMET: síndrome metabólica; IMC: índice de massa corporal em $\mathrm{kg} / \mathrm{m}^{2}$; CC: circunferência da cintura em $\mathrm{cm}$; EST.HEP: esteatose hepática não-alcoólica. A) TRIGLI em voluntários com e sem SÍNMET. * $\mathrm{p} \leq 0.05$; B) PAS em voluntários com e sem SÍNMET. *p $\leq 0.01$; C) PAD em voluntários com e sem SÍNMET. * $\mathrm{p} \leq 0.05$; D) HDL-C em voluntários com e sem SÍNMET. *p $\leq 0.01$; E) IMC em voluntários com e sem EHNA. * $\mathrm{p} \leq 0.01$; F) CC em voluntários com e sem EHNA. *p $\leq 0.05$. Todas as relações foram feitas pelo teste de correlação de Pearson.

estudo, no qual não houve diferença significativa entre o IMC dos adolescentes, pós-púberes, do sexo masculino e do feminino.

A obesidade em crianças e adolescentes está fortemente associada com o aumento do risco de doenças cardiovasculares prematuras, SM e EHNA, levando a uma ampliação da taxa de morte prematura nessa população $0^{(29,31,32,35)}$. Sendo assim, o presente estudo está em consonância com a literatura, pois mais de $50 \%$ dos adolescentes obesos apresentaram alta ocorrência de fatores 
de risco cardiovasculares como CC, PAS, LDL-C acima dos valores de referência e HDL-C abaixo dos valores preconizados, além de uma grande ocorrência de EHNA e SM.

O fator de risco cardiovascular mais presente nos adolescentes deste estudo foi a circunferência da cintura, onde $100 \%$ dos adolescentes apresentavam valores acima do ponto de corte preconizado pela $\operatorname{IDF}^{(23,24)}$. Os valores percentuais encontrados apresentam-se superior aos 55\% encontrados por pesquisadores de outro estudo também realizado em adolescentes brasileiros ${ }^{(36)}$. A porcentagem encontrada de circunferência da cintura acima dos valores de corte é preocupante, pois sugere que pelo menos no sexo masculino, altos valores da circunferência da cintura em adolescentes predizem precocemente a SM em adultos ${ }^{(37)}$.

Os achados da presente pesquisa não demonstraram uma ocorrência significativa de hiperglicemia de jejum $(8,8 \%)$. No entanto, esse dado não foi encontrado apenas no presente estudo ${ }^{(13,14,36)}$. Em outros estudos com adolescentes, os autores relataram que a hiperglicemia de jejum foi o fator de risco menos frequente ${ }^{(13,14)}$. Outro estudo realizado no Brasil apresentou uma ocorrência (2\%) de hiperglicemia de jejum como fator de risco, porcentagem ainda menor do que a encontrada nesta pesquisa ${ }^{(36)}$.

A ocorrência de EHNA (76,5\%) encontrada no estudo em questão foi maior que a apresentada na literatura para adolescentes obesos $(38 \%)^{(5)}$. No entanto, outro estudo ${ }^{(38)}$ também encontrou uma alta ocorrência de EHNA. Em uma amostra de 861 crianças e adolescentes obesos, 587 (68,2\%) apresentaram EHNA.

Essa alta ocorrência de EHNA na população estudada remete a uma situação preocupante, uma vez que indivíduos com EHNA apresentam taxa de mortalidade por qualquer motivo, de $34 \%$ a $86 \%$ maior que a população geral. Uma das principais causas de morte são as doenças cardiovasculares $^{(4)}$, por estarem relacionadas com os fatores que predizem o desenvolvimento de doença coronariana, dislipidemia, obesidade central e $\mathrm{SM}^{(38)}$.

Outro fato preocupante dos achados foi que a síndrome metabólica esteve presente em $50 \%$ dos nossos voluntários, acima dos $38,7 \%$ descritos para a população pediátrica geral $^{(13)}$, e acima da ocorrência de $25,7 \%$ encontrada em crianças e adolescentes asiáticos ${ }^{(38)}$. População essa que os parâmetros de circunferência da cintura são utilizados como referência para população sul-americana na caracterização da $\mathrm{SM}^{(23,24)}$.

Observou-se também uma alta ocorrência concomitante EHNA com SM (53,8\%). A ocorrência de EHNA juntamente com SM encontrada no estudo foi similar à observada em um estudo norte americano, no qual os pesquisadores relataram que 50\% das crianças e adolescentes com EHNA apresentaram $\mathrm{SM}^{(3)}$.
De fato foi visto correlação entre SM, EHNA e fatores de risco cardiovascular. Este achado está em consonância com estudo que demonstrou que a presença de esteatose hepática pode ser considerada a manifestação hepática da $\mathrm{SM}^{(10)}$.

Ademais, notou-se maior ocorrência de EHNA (78,6\%) e SM $(64,3 \%)$ no sexo masculino em relação aos $75 \%$ de EHNA e $40 \%$ de SM no sexo feminino. Outro estudo brasileiro realizado em adolescentes também encontrou uma maior ocorrência de EHNA no sexo masculino $(19,5 \%)$ em relação ao sexo feminino $(13 \%)^{(39)}$, porém com uma diferença percentual bem menor do que a encontrada no presente estudo.

Em relação à maior ocorrência de SM no sexo masculino, pesquisa com adolescentes norte-americanos utilizando os critérios da International Diabetes Federation para identificação da SM, mesmos critérios utilizados no presente estudo, também encontraram maior incidência no sexo masculino, entretanto a ocorrência foi de $6,7 \%$ no sexo masculino e de $2,1 \%$ no sexo feminino ${ }^{(40)}$, muito menor que a encontrada neste estudo.

$\mathrm{Na}$ mesma época do estudo realizado nos EUA, pesquisadores brasileiros identificaram diferenças na ocorrência de SM entre os sexos. As porcentagens encontradas nos adolescentes brasileiros foram 34,8\% para o sexo masculino e $15,6 \%$ para o feminino ${ }^{(11)}$. A porcentagem observada nos adolescentes brasileiros foi maior que a encontrada nos EUA, porém ainda menor do que a identificada no estudo em questão.

Recentemente, pesquisa com adolescentes brasileiros encontrou valores similares aos deste estudo. Os pesquisadores verificaram uma ocorrência $49,1 \%$ de SM no sexo masculino, no entanto, a ocorrência $(26,9 \%)$ no sexo feminino $^{(14)}$ ainda foi menor que o percentual encontrado neste estudo.

As relações fisiológicas entre a maior ocorrência de EHNA e SM em adolescentes do sexo masculino ainda não são descritas pela literatura. Porém, acredita-se que essa maior ocorrência de EHNA e SM em adolescentes, póspúberes, do sexo masculino esteja fortemente relacionada com a diferença topográfica da distribuição de gordura corporal entre os sexos e com a influência dos hormônios sexuais.

O presente estudo teve como limitação o tamanho da amostra, sendo necessários estudos com maior número de indivíduos para confirmar os achados na população estudada.

No entanto, torna-se relevante diante da grande possibilidade, de $50 \%$ a $70 \%$, dos adolescentes obesos permanecerem obesos na vida $\operatorname{adulta}^{(28)}$. Fazendo-se necessário efetivar estratégias terapêuticas, preventivas, 
educativas e de promoção da saúde nessa população, uma vez que a EHNA, a SM, as doenças cardiovasculares e a obesidade são doenças preveníveis com a realização de atividade física e alimentação saudável.

\section{CONCLUSÃO}

Foi observada uma alta ocorrência de EHNA, SM e fatores de risco cardiovascular nos adolescentes obesos, com uma maior incidência no sexo masculino. Analisou-se também que a EHNA e a SM se relacionaram com fatores de risco cardiovascular como índice de massa corporal, circunferência da cintura, triglicerídeos, hipertensão arterial sistólica e diastólica, e com baixos valores de HDL-C.

Constatou-se também uma forte correlação entre EHNA com a SM e FRCs, pois os adolescentes obesos com EHNA apresentaram maior chance de desenvolver SM e doenças cardiovasculares, sugerindo a necessidade de intervenções multiprofissionais.

\section{REFERÊNCIAS}

1. Chalasani N, Younossi Z, Lavine JE, Diehl AM, Brunt EM, Cusi K, et al. The Diagnosis and Management of Non-Alcoholic Fatty Liver Disease: Practice Guideline by the American Association for the Study of Liver Diseases, American College of Gastroenterology, and the American Gastroenterological Association. Hepatoloty. 2012;55(6):2005-23.

2. Tiniakos DG, Vos MB, Brunt EM. Nonalcoholic Fatty Liver Disease: Pathology and Pathogenesis. Ann. Rev Pathol Mech Dis. 2010;5:145-171.

3. Schwimmer JB, Pardee PE, Lavine JE, Blumkin AK, Cook S. Cardiovascular Risk Factors and the Metabolic Syndrome in Pediatric Nonalcoholic Fatty Liver Disease. Circulation. 2008;118(3):277-83.

4. Perazzo H, Poynard T, Dufour JF. The Interactions of Nonalcoholic Fatty Liver Disease and Cardiovascular Diseases. Clin Liver Dis. 2014;18(1):233-48.

5. Feldstein AE, Charatcharoenwitthaya P, Treeprasertsuk S, Benson JT, Enders FB, Angulo P. The natural history of non-alcoholic fatty liver disease in children: a followup study for up to 20 years. Gut. 2009;58(11):1538-44.

6. Erickson SK. Nonalcoholic fatty liver disease. J Lipid Res. 2009;50(Suppl):S412-S416.

7. Ferolla SM, Ferrari TC, Lima ML, Reis TO, TavaresJr WC, Couto OF, et al. Dietary patterns in Brazilian patients with nonalcoholic fatty liver disease: a crosssectional study. Clinics (Sao Paulo). 2013;68(1):11-17.
8. Cotrim HP, Parise ER, Oliveira CP, Leite N, Martinelli A, Galizzi J, et al. Nonalcoholic fatty liver disease in Brazil. Clinical and histological profile. Ann Hepatol. 2011;10(1):33-7.

9. Souza FIS, Amâncio OMS, Sarni ROS, Pitta TS, Fernandes AP, Fonseca FLA, et al. Doença hepática gordurosa não alcoólica em escolares obesos. Rev Paul Pediatr. 2008;26(2):136-41.

10. Soler GLN, Silva AWSM, Silva VCG, Teixeira RJ. Doença hepática gordurosa não-alcoólica: associação com síndrome metabólica e fatores de risco cardiovascular. Rev. SOCERJ. 2008;21(2):94-100.

11. Caranti DA, Lazzer S, Dâmaso AR, Agosti F, Zennaro R, de Mello MT, et al. Prevalence and risk factors of metabolic syndrome in Brazilian and Italian obese adolescents: a comparison study. Int J Clin Pract. 2008;62(10):1526-32.

12. Angurana SK, Angurana RS. Prevalence of metabolic syndrome in adolescents: Reason to worry. Indian J Endocrinol Metab. 2013;17(6):1135-6.

13. Moraes ACF, Fulaz CS, Oliveira Netto ER, Reichert FF. Prevalência de síndrome metabólica em adolescentes: uma revisão sistemática. Cad Saúde Pública. 2009;25(6):1195-202.

14. Campos L de A, Amancio OMS, Costa e Forti A. Impact of obesity on metabolic syndrome in Brazilian adolescents - a population-based study. Diabetes Care. 2013;36(9):e139-40.

15. Stanley TL, Chen ML, Goodman E. The typology of metabolic syndrome in the transition to adulthood. J Clin Endocrinol Metab. 2014;99(3):1-9.

16. Instituto Brasileiro de Geografia e Estatística - IBGE. Pesquisa de Orçamentos Familiares - POF 2008-2009. Comunicação Social 27 de agosto de 2010 [acesso em 2014 Jan 4]. Disponível em: http://saladeimprensa. ibge.gov.br/index.php?option $=$ com_saladeimprensa\& view $=$ noticias $\& \mathrm{id}=1 \&$ paginar $=1 \&$ quantidade $=10 \& \mathrm{pa}$ gina $=1 \&$ busca $=1 \&$ Itemid $=1719 \&$ data-inicio $=\&$ data-fi $\mathrm{m}=\&$ alvo $=$ obesidade + na + adolescencia .

17. Kuczmarski RJ, Ogden CL, Guo SS, GrummerStrawn LM, Flegal KM, Mei Z, et al. 2000 Centers for Disease Control (CDC) growth charts for the United States: methods and development. Vital Health Stat. 2002;11(246):1-190.

18. Marshall WA, Tanner JM. Variations in pattern of puberal changes in girls. Arch Dis Childh. 1969;44(233):291-303. 
19. Marshall WA, Tanner JM. Variations in pattern of puberal changes in boys. Arch Dis Childh. 1970;45(239):13-23.

20. Moll AJ. Esteatose hepática: avaliação por ultrasonografia. Informativo Med D’Or (RJ). 2006;6:67 .

21. Saadeh S, Younossi ZM, Reme EM, Gramlich T, Ong JP, Hurley M, et al. The utility of radiological imaging in nonalcoholic fatty liver disease. Gastroenterol. 2002;123(3):745-50.

22. Diniz ALD, Tomé RAF, Debs CL, Carraro R, Roever LB, Pinto RMC. Avaliação da reprodutibilidade ultrassonográfica como método para medida da gordura abdominal e visceral. Radiol Bras. 2009;42(6):353-7.

23. Zimmet P, Alberti G, Kaufman F, Tajima N, Silink M, Arslanian S, et al. The metabolic syndrome in children and adolescents: the IDF consensus. Diabetes Voice. 2007;52(4):29-32.

24. Zimmet P, Magliano D, Matsuzawa Y, Alberti G, Shaw J. The Methabolic Syndrome: A Global Public Health Problem and A New Definition. J Athrosclerosis Thrombosis. 2005;15(6):295-300.

25. V Diretrizes Brasileiras de Dislipidemias e Prevenção da Aterosclerose. Arq Bras Cardiol. 2013;101(4Supl.1):122.

26. International Diabetes Federation - IDF. The IDF consensus worldwide definition of the metabolic syndrome [acesso em 2014 Jan 10]. April 14, 2005. Disponível em: http://www.idf.org/metabolicsyndrome

27. Alberti KGMM, Zimmet P, Shaw J. Metabolic syndrome: a new world-wide definition. A Consensus Statement from the International Diabetes Federation. Diabetic Medicine. 2006;23(5):469-80.

28. Associação Brasileira para o Estudo da Obesidade e da Síndrome Metabólica - ABESO. Diretrizes brasileiras de obesidade 2009/2010 [acesso em 2014 Jan 20]. Disponível em: http://www.abeso.org.br/pdf/ diretrizes_brasileiras_obesidade_2009_2010_1.pdf.

29. Van Grouw J, Volpe SL. Childhood obesity in America. Current Opinion in Endocrinology, Diabetes \& Obesity. 2013;20(5):396-400.

30. Travi MIC, Bastos PRHO, Pontes ERJC. Prevalência de sobrepeso, obesidade e circunferência abdominal alterada em escolares de 6 a 11 anos de idade em Campo Grande/MS. Rev Bras Promoç Saúde. 2011;24(1):5462.
31. Balakrishnan PL. Identification of Obesity and Cardiovascular Risk Factors in Childhood and Adolescence. Pediatr Clin North Am. 2014;61(1):15371.

32. Franks PW, Hanson RL, Knowler WC, Sievers ML, Bennett PH, Looker HC. Childhood obesity, other cardiovascular risk factors, and premature death. $\mathrm{N}$ Engl J Med. 2010;362(6):485-93.

33. Corey KE, Kaplan LM. Obesity and liver disease: the epidemic of the twenty-first century. Clin Liver Dis. 2014;18(1):1-18.

34. Ramos MLM, Pontes ERJC, Ramos MLM, Barros VRSP. Sobrepeso e obesidade em escolares de 10 a 14 anos. Rev Bras Promoç Saúde. 2013;26(2):223-32.

35. Yilmaz Y, Younossi ZM. Obesity-Associated Nonalcoholic Fatty Liver Disease. Clin Liver Dis. 2014;18(1):19-31.

36. Rizzo AC, Goldberg TB, Silva CC, Kurokawa CS, Nunes HR, Corrente JE. Metabolic syndrome risk factors in overweight, obese, and extremely obese Brazilian adolescents. Nutr J. 2013;12(19):1-9.

37. Barzin M, Asghari G, Hosseinpanah F, Mirmiran P, Azizi F. The association of anthropometric indices in adolescence with the occurrence of the metabolic syndrome in early adulthood. Pediatr Obes. 2013;8(3):170-7.

38. Fu JF, Shi HB, Liu LR, Jiang P, Wang CL, Liu XY. Non-alcoholic fatty liver disease: An early mediator predicting metabolic syndrome in obese children? World J Gastroenterol. 2011;17(6):735-42.

39. Fernandes MTB, Ferraro AA, Azevedo RA, Fagundes Neto U. Metabolic differences between male and female adolescents with non-alcoholic fatty liver disease, as detected by ultrasound. Acta Paediatrica. 2010;99(8):1218-23.

40. Ford ES, Li C, Zhao G. Prevalence of the Metabolic Syndrome Among U.S. Adolescents Using the Definition From the International Diabetes Federation. Diabetes Care. 2008;31(3):587-9.

\section{Endereço para Correspondência:}

Nadia Carla Cheik

Faculdade de Educação Física / UFU

Rua Benjamin Constant, 1286

Bairro Aparecida

CEP: 38400-678 - Uberlândia - MG - Brasil

e-mail: wener_educa@hotmail.com 